\title{
Is gravity, the curvature of spacetime or a quantum phenomenon?
}

\section{Alfonso Leon Guillen Gomez}

\author{
Independent scientific researcher, 127A Street 53A68 Flat 514, Bogota, Colombia \\ aguillen@gmx.net
}

\begin{abstract}
Gravity is the curvature of spacetime, the structural property of static gravitational field, a geometric field, in curved coordinates, according the functions guv, that express geometric relations between material events. Course, general relativity is a relational theory, however, gravity, a thinking category, has symetric physical effects with matter. We use, analitic and critic method of reread the general relativity, since the perspective of the history of the science and the philosophy of the science. Our goal is driver the debate on gravity, to the arena of the quantum physics, but without the ballast of the general relativity. We find that through of relativist aether was attempted transform spacetime in a substantia without succes, the consequence was return to problematic geometric field. The philosophy of the science intervenes, and according the best philosophical theory of substantivalism, spacetime is a inmaterial, geometric substantia. Then, the metaphysics arrives to a full solution in the super-substantivalism theory, that affirms: matter arises from geometric spacetime. Thus, it explains consistently the symetric physical effects between spacetime and matter. Surely, this solution is a medieval speculation. Our conclusion is that since general relativity do not defined physically spacetime leads necessarily to philosophical definitions of relationism and substantivalism on spacetime that are unacceptable physically. Therefore, gravity is not the curvature of spacetime.
\end{abstract}

Keywords: gravity, spacetime, aether, vacuum, physics, metaphysics, quantum physics

PACS: 03. Quantum mechanics, field theories, and special relativity, 03.50.-z Classical field theories, 03.65.-w Quantum mechanics, 04. General relativity and gravitation, 04.20.-q Classical general relativity

\section{Council for Innovative Research}

Peer Review Research Publishing System

Journal: Journal of Advances in Physics

Vol 4, No. 1

editor@cirworld.com 


\section{INTRODUCTION}

Gravity in general relativity is the curvature of space time. But, what is spacetime?. Both, general relativity and aristotle's philosophical school declare the contingent existence of spacetime with relation to matter, in relativity, and to aether, in Aristotle. The idea of aether between the medieval and current period had a profound development, in philosophy and in science. The scientific result is that the primitive substance of the aether passes to luminiferous and gravitational aether, that was substituted for the electromagnetic aether and, this was replaced for the relativistic aether, finally discarded by the field. Although, the static gravitational field, in a unknown and unacceptable hypothesis of Einstein, would be a energetic field, the fact is that according to the Einstein's equations, the static gravitational field is a geometric field and spacetime, its structural property, course general relativity is a relationist theory. However, various physical considerations point out that spacetime must be a substantia, that valid the conception of the substantivalist interpretation of spacetime rival of the relationist theory; both theories were originated in the philosophical medieval discussion between Newton and Leibniz. The current solution of the philosophy and metaphysic declares the spacetime as inmaterial substance inside the context of general relativity, i.e. from geometric nature, with symetric physical effects between spacetime and matter. How can spacetime have physical effects?. The metaphysic super substantivalism says, because matter arises of spacetime and, last instance, also matter is geometry. Both, relationist and substantivalist theories affirm spacetime is of geometric nature; but, while for relationist theory, spacetime is a thinking category, in change, spacetime is real to substantivalism. Therefore, surely both philosophic solutions must be wrong.

We reject spacetime as substantia, since the naked spacetime does not exist and consequently it has contingent existence. Due, to which in the nature only exist, the vacuum, matter and radiation (forms of material existence), necessarily, the spacetime must be, in the sense original of field, a internal state (like a property) of matter and gravity is not phenomenon of spacetime. Thus, gravity must be a material phenomenon and the most appropriate hypothesis: gravity is a quantum phenomenon, independent of the spacetime. This is the hypothesis induced, and that will be proposal in this work.

\section{HISTORICAL DEVELOPMENT OF THE AETHER CONCEPT}

Although, aether was a recurring idea at thinking on the Universe among the ancients, in the third century B.C, formally was introduced, against the philosophical thesis of atomism of Leucippus and Democritus on atoms and the vacuum, via cosmology of the speculative philosophy-physics, due to a ontological reason, by Aristotle, at region beyond of the Moon, like substance weightless, filling the space, fifth essence indestructible and unchanging, unlike of the others four substances of water, air, fire and earth convertible each one in other; i.e, aether like the substance that fills the vacuum, because according to Aristotle there is no vacuum and to aristotelians: nature abhors vacuum (Aristotle, 1930), that medieval thinkers did famous as: horror vacui. This aether is of mechanic character, vision active during a long time, more of twenty one centuries. With Renascence the concept of ether, gradually left the arena of philosophy, passing more to the arena of physics. In 1644, Descartes proposed aether as a continuous, made up of very small particles, transmitting forces from one object to another by collisions of the particles, that completely fills the space not occupied by solid bodies because there is not true vacuum; it is contrary to reason say that there is a vacuum or space in which there is absolutely nothing (Descartes, 1969), i.e, due to a logical reason and since the action of forces is by contact, there is no action to distance, this time, due to a physical reason. Also, in 1644, Torricelli realized their experiments that proved the existence of the vacuum and that the effects, attributed to the horror vacui, were actually due to the air pressure, encouraging the revival of atomism and scientific revolution that, in 1604, mirabilis year, Galilei had inaugurated, with the introduction of the method observation-experiment-induction (Gower, 2002), but despite Torricelli's experiments the discussion between the plenists (no vacuum is possible in nature) and the vacuists (vacuum is possible) was continued (Jousten, 2008). However, in 1678 and 1690, in analogy with sound, Huygens presented its theory ondulatory of the light, second physical reason to luminiferous aether, like medium of propagation of this wave, and he said on particles of aether, between which act mechanical forces, they are hard elastic things which transmitted impulses, without being themselves displaced, and different to gravitational aether which circulates around the centre of the Earth, cause of gravity, its third physical reason. In 1687, when Newton formulated, at Mathematical Principles of Natural Philosophy, the laws of motion from Galilei the vacuum became a essential component of the Universe, it introduced the action to distance instant, and to conserve the principle of relativity and the energy-momentum conservation law, the Galileo-Newton vacuum must be assumed to be absolutely empty (Wang, 2007), but Newton it contradicted, in 1704, in his paper on Opticks and at its updated second edition, where he postulated a real aether, as luminiferous aether to explain refraction and diffraction of light. In 1717 , Newton declared that aether (gravitational aether) is a stationary tenuous medium, compound by particles, with a variable density, most denser in empty space than in the vicinity of massive bodies, to explain gravitational effects (Newton, 1730); surely, the introduction of vacuum, in the classical physics did not eliminates initially to the aether but it became its dangerous rival. In 1748, Le Sage proposed an aether consisting of tiny particles, called corpuscles, flowing at all direction with enormous speed. In 1801, Young said aether is a gas in absolute rest. Due, to discovery of the polarization of light, in 1817, Fresnel introduced, transverse wave theory of light and, then, Young proposed a periodic transverse displacement of aether particles. According Fresnel, the aether became solid-like and rigid, that however, yet allowed the free passage of heavenly bodies, while aether flowed through the interstices of material bodies even on the smallest scale, but without that matter has a small dragging effect on aether; the density of aether in a material body was different than in the free aether. Between 1828 and 1839, Cauchy proposed an aether dynamic, due to its changes in its density and in its elasticity, in consequence, aether is contractile or labile, possessing a negative compressibility (today called a 
negative $\wedge$ ). Green pointed out that Cauchy's contractile aether would be unstable, tending to shrink its size, all the time. In 1845, Stokes coincided partially with Fresnel, since, aether flows almost unhindered through all matter, whose implication is that aether flows through mass of bodies, but the matter in motion of rotation or translation drags aether, therefore, the Earth drags the aether. In decade of 1860, Maxwell formulated the electromagnetic aether, having the properties specified by its equations (Eddington, 1938), as a quasi-material elastic stationary medium, as the preferred frame of reference in which light propagates with constant speed in all directions; whether aether is continuous or discrete remained undecided. With the discovery done, in 1887, by Michelson-Morley, of the constant speed of light, in 1889 and 1891, FitzGerald, proposed that the forces binding the molecules of a solid are modified by motion of the solid through aether in such way that the dimension of the arm of the interferometer, in Michelson-Morley's experiment, would be shortened in the direction of motion and that this contraction neutralizes the optical effect sought; aether not only changes the course of objects (as gravitational aether does), also aether changes the size of objects; in consequence, aether produces the relativistic effect of contraction the length of any object, this contraction occurring in the direction of motion and in proportion to the speed through aether. In 1895, Lorentz improved FitzGerald's hypothesis, also he improved Maxwell's electromagnetic aether by the immobile special frame, where are valids the laws of electrodynamic; given that the atoms of all solids are held together by electrical forces; thus the motion of a body, according to Maxwell's mechanic, superposes upon the electrostatic forces between the atoms a magnetic effect due to the motion; result would be a contraction of the body in the direction of motion which is proportional to the square of the ratio of the translation speed and of light speed and which would have a magnitude such as to annul the effect of aether-drift, in Michelson-Morley's experiment; this length contraction leads to time dilation for all phenomena that obey Newton's laws and/or Maxwell's laws, in inertial frames, and both contractions lead to the Lorentz's transformation between inertial frames, that replaced Galilei's transformation; this theory was later confirmed, whenever the experiment is performed in vacuum, the aether effect on the optical interferometer is totally annulled. (Ranzan, 2010, Stokes, 1845 and Haldane, 2011).

The evolution of aether concept drove to that, as electromagnetic aether constitutes the carrier substrate of the electromagnetic wave and provides the special frame in which Maxwell's equations are valids and, as gravitational aether, an anachronistic concept after of Maxwell, i.e, luminiferous aether, acts as a interaction force on particles and bodies, carries the action in contact and produces the gravitational effect.

Newton, at his corpuscular theory, explained the propagation of light supported in aether. Huygens, Young and Maxwell were accord partially with he. But, they corrected and added that aether is the medium of propagation of the light wave (the electromagnetic wave of Maxwell), thus, aether becomes physically necessary; Newton said that light rays consisted of a stream of particles in rectilinear motion and that the light particles stimulated, or were accompanied by, vibrations in an all pervading aether. Huygens formulated the wave theory of light in which waves propagated longitudinally, with a speed finite, through a stationary aether, also, Euler and Young were accord. Maxwell said, that light is an electromagnetic wave, concept originated at Faraday's lines of electrical and magnetic forces. On lines of force, Maxwell said, were tubes of aether rotating on their axes. The centrifugal force, of such rotations, causes the tubes to expand sideways and contract lengthways, as Faraday had suggested in order to explain electric-magnetic attraction and repulsion. These rotating tubes carry electrical particles along, from one tube to the next and the next, in what amounts to a form of transverse undulations at the speed of light. Notwithstanding the inclusion of microscopic rotating tubes, aether was viewed as a stationary medium. (Ranzan, 2010). Electrodynamics was visualized like mechanics of fluids, thus, the aether was visualized as water at rest, as a medium that permeates the universe and enables electro-magnetic waves to propagate (Dannon, 2011).

Descartes, Newton, Huygens, Le Sage and Laplace explained gravity from aether. To Descartes and Huygens the aether is a sea, of vortexes in motion, carrying the bodies and that produces the gravitational effects; to Newton caused by impulses of a stream of aether particles, hitting bodies or by variations in an all-pervading aether. Newton, in repeated attempts to unify various branches of physics, led him to the concept of wave/particle duality and to model of gravity in which the gravitational field could be described as a density gradient, and in which the deflection of light or matter by the field was modelled as the effect of a variation in refractive index. In singly-connected space, this approach can be topologically equivalent to a curved-space model of gravity while, general relativity is a curved spacetime model of gravity (Baird, 2000), but, due to that this explication of gravity was not included in Principia, it is not recognized as part of his work on physics. Le Sage used its corpuscles as the basis for a kinetic theory of gravity. Laplace declared that the density of the aether is proportional to the radial distance from the center of a body and that the force of gravity is generated by the impulse of such aether medium, also he said that the effect of gravity is propagated with a speed between 7-million and 100-million times that of light; but, this rules out the notion that the flow of the medium itself is involved in cause of gravity. (Ranzan, 2010).

With the gradual pass of aether from the arena of the philosophy into the arena of the physics, aether like substrate of the electromagnetic wave propagating transversely with a ultra-high speed, and that aether also produces gravitational effects, the ether would have ridiculously mysterious nature and mechanical properties, such as be massive, weightless, stationary super fluid, also with very high speed, gelatinous with very high elasticity, very high rigidity, incompressible, and to lack totally of resistance to motion of bodies, ie, with contradictory properties, physically absurd and with an impossible real existence.

When Maxwell's electrodynamics arises the luminiferous aether is replaced by the electromagnetic aether that Maxwell still tried to interpret his field theory mechanically by means of mechanical ether models. But these attempts receded gradually to the background following the representation -purged of any unnecessary additions- by Heinrich Hertz, so that, in this theory the field finally took the fundamental position which had been occupied in Newton's mechanics by the material points. At first, however, this applies only for electromagnetic fields in empty space. In its initial stage the theory was yet quite unsatisfactory for the interior of matter, because there, two electric vectors had to be introduced, which were 
connected by relations dependent on the nature of the medium, these relations being inaccessible to any theoretical analysis. An analogous situation arose in connection with the magnetic field, as well as in the relation between electric current density and the field (Einstein, 1936). Also, every attempt to explain the electromagnetic phenomenon in moving, with the help of the motion of the ether, motion through the ether, or both these movements, proved unsuccessful (Einstein and Infeld, 1938).

\section{RELATIVISTIC AETHER}

Previous to special relativity, surely, Einstein considered aether as a big mistake of the classical physics, since, he rejected aether as material substance, as later, he stated repeatedly.

In the special relativity, Einstein resolved the main problem of epoch, on the relativity of the motion. He unified the theories of Galilei-Newton and Maxwell, and finished with the special frame in which Maxwell's equations were valids, one of the three physical foundations according classical mechanics, of the existence of aether. This goal was reached by Einstein, applying principle of relativity of Galilei, generalized to electrodynamics and optics, i.e, Galilei's theory on bodies in inertial motion, unified with Maxwell's theory for stationary bodies, and principle that electromagnetic wave is always propagated, at any direction, in vacuum with a constant velocity $c$. This first success allowed to Einstein, to say that: luminiferous ether will prove to be superfluous inasmuch as the view here to be developed will not require an absolutely stationary space provided with special properties, nor assign a velocity-vector to a point of the empty space in which electromagnetic processes take place (Einstein, 1905a). With a new argument, Einstein reaffirmed, is not required a special frame given that: the relativity principle states that all natural laws that hold in a reference frame $\mathrm{K}^{\prime}$ moving uniformly relative to the ether are identical with those that hold in $\mathrm{K}$, a reference frame at rest relative to the ether. If that is so, we can just as well imagine the ether is at rest relative to $\mathrm{K}^{\prime}$, not $\mathrm{K}$. It is completely unnatural to distinguish the two reference frames $\mathrm{K}^{\prime}$ and $\mathrm{K}$ by introducing an ether that is at rest in one. A satisfying theory can only be reached if we dispense with the ether hypothesis. (Einstein, 1909).

Inside the set of papers, that Einstein wrote between 1905 (Einstein, 1905b) and 1917 (Einstein, 1917), he formulated progressively its theory of the duality wave-particle of light completely. Thus, Einstein, in 1909, attacked explicitly the main and most old of the three physical foundations of aether as carrier substrate of the electromagnetic wave, with that he obtained its second success, when he advanced that also light can be a emission of particles, supported in the equivalence between mass and energy (Einstein, 1905c), in such case is unnecessary an propagation medium. Einstein said: we regard the ether hypothesis as obsolete. In effect: a large body of facts shows undeniably that light has certain fundamental properties that are better explained by Newton's emission theory of light than by the oscillation theory. For this reason, I believe that the next phase in the development of theoretical physics will bring us a theory of light that can be considered a fusion of the oscillation and emission theories... The foundation of the ether hypothesis is the experimentally based assumption that the ether is at rest (false assumption due to relativity principle, author)... then the electromagnetic fields that make up light no longer appear as a state of a hypothetical medium, but rather as independent entities that the light source gives off, just as in Newton's emission theory of light... the inertial mass of an object is diminished by the emission of light. The energy given up was part of the mass of the object. One can further conclude that every absorption or release of energy brings with it an increase or decrease in the mass of the object under consideration. Energy and mass seem to be just as equivalent as heat and mechanical energy... Relativity theory has changed our views on light. Light is conceived not as a manifestation of the state of some hypothetical medium, but rather as an independent entity like matter. Moreover, this theory shares with the corpuscular theory of light the unusual property that light carries inertial mass from the emitting to the absorbing object... Planck's theory leads to the following conjecture. If it is really true that a radiative resonator can only assume energy values that are multiples of hv, the obvious assumption is that the emission and absorption of light occurs only in these energy quantities. On the basis of this hypothesis, the light-quanta hypothesis, the questions raised above about the emission and absorption of light can be answered. As far as we know, the quantitative consequences of this light-quanta hypothesis are confirmed. (Einstein, 1909).

The third and last physical foundation of aether, as carrier of the gravitational action in contact, was not considered by Einstein, due to that special relativity can not assume the presence of gravitational fields, since it is not possible to introduce the Newton's equations of the gravity, given that its instantaneous action violates the speed c, maximum limit. Years later, it was changed the gravitational potential, to a scalar, after to a vector, and finally, to a symmetric tensor, but the results are not consistent with the experimental observations (Guillen, 2010).

Einstein after that published the final version of the general relativity (Einstein, 1916), where according to general covariance, he exposed spacetime's concept relationist, i.e, as relational ideal geometric object, completely lack of physical properties, but in June of that year, he changed unexpectedly at a interchange of letters with Lorentz, identifying spacetime with the relativistic aether, a new concept of gravitational relativist aether, totally strange to electromagnetic aether that he had rejected, this hipothesis was presented formally in 1918 (Cassini and Levinas, 2009).

Author believes probable, Einstein was motivated by explain the action, in contact, that was not included in special relativity, since it does not apply, due to that the photon-electromagnetic wave possess energy and momentum, allowing it acts by contact, but fundamental to general relativity, since that is a theory on gravity. Whether well gravity is a geometric field, its action in contact needs a geometric medium with physical properties, although not is a material medium. Such physical properties are derived from that can not exist a space absolutely empty, since it will be occupied always by a gravitational field, in this sense by gravitational aether, thus Einstein said: while according to the special theory of relativity a part of space without matter and without electromagnetic field seems to be characterized as absolutely empty, e. g. not characterized by any physical quantities, empty space in this sense has according to the general theory of relativity physical qualities which are mathematically characterized by the components of the gravitational potential, that determine 
the metrical behavior of this part of space as well as its gravitational field. One can quite well construe this circumstance in such a way that one speaks of an aether, whose state of being is different from point to point. Only one must take care not to attribute to this aether properties similar to properties of matter (for example every point a certain velocity) (Einstein, 1918).

In 1920, Einstein presented, in a conference, in the University of Leyden, its theory on the relativistic gravitational aether. He emphasized on the need for relativistic ether to remove action to distance, but other than a mechanical ether is not independent of matter, neither any form of it since that is a geometric real being. The relativistic ether is determined by matter that simultaneously determines it. The relativistic ether is another denomination of spacetime, that can not exist without gravitational field. Matter and spacetime are two different real beings, but spacetime exists only as gravitational field and, in consequence, depending of existence of matter, since in its absence does not exist gravitational field, i.e curvature. Therefore, with the introduction relativistic gravitational ether, Einstein left the relationist model of spacetime and adopted the substantialist model of spacetime, i.e the spacetime is a geometric object with a real existence although is a contingent existence. He said: How does it come about that alongside of the idea of ponderable matter, which is derived by abstraction from everyday life, the physicists set the idea of the existence of another kind of matter, the aether? The explanation is probably to be sought in those phenomena which have given rise to the theory of action at a distance.. assuming that the Newtonian action at a distance is only apparently immediate action at a distance, but in truth is conveyed by a medium permeating space, whether by movements or by elastic deformation of this medium. Thus the endeavour toward a unified view of the nature of forces leads to the hypothesis of an aether... inertial resistance opposed to relative acceleration of distant masses presupposes action at a distance; and as the modern physicist does not believe that he may accept this action at a distance, he comes back once more, if he follows Mach, to the aether, which has to serve as medium for the effects of inertia. But this conception of the aether to which we are led by Mach's way of thinking differs essentially from the aether as conceived by Newton, by Fresnel, and by Lorentz. Mach's aether not only conditions the behaviour of inert masses, but is also conditioned in its state by them... Mach's idea finds its full development in the aether of the general of relativity. According to this theory the metric qualities of the continuum of spacetime differ in the environment of different points of spacetime, and are partly conditioned by the matter existing outside of the territory under consideration. This space-time variability of the reciprocal relations of the standards of space and time, or, perhaps, the recognition of the fact that "empty space" in its physical relation is neither homogeneous nor isotropic, compelling us to describe its state by ten functions (the gravitation potentials), has, I think, finally disposed of the view that space is physically empty. But therewith the conception of the aether has again acquired an intelligible content, although this content differs widely from that of the aether of the mechanical undulatory theory of light. The aether of the general theory of relativity is a medium which is itself devoid of all mechanical and kinemassic qualities, but helps to determine mechanical (and electromagnetic) events... What is fundamentally new in the aether of the general theory of relativity as opposed to the aether of Lorentz consists in this, that the state of the former is at every place determined by connections with the matter and the state of the aether in neighbouring places, which are amenable to law in the form of differential equations; whereas the state of the Lorentzian aether in the absence of electromagnetic fields is conditioned by nothing outside itself, and is everywhere the same... I think, that the aether of the general theory of relativity is the outcome of the Lorentzian aether, through 'relativation'... If we consider the gravitational field and the electromagnetic field from the standpoint of the aether hypothesis, we find a remarkable difference between the two. There can be no space nor any part of space without gravitational potentials; for these confer upon space its metrical qualities, without which it cannot be imagined at all. The existence of the gravitational field is inseparably bound up with the existence of space. On the other hand a part of space may very well be imagined without an electromagnetic field; thus in contrast with the gravitational field, the electromagnetic field seems to be only secondarily linked to the aether, the formal nature of the electromagnetic field being as yet in no way determined by that of gravitational aether... Since according to our present conceptions the elementary particles of matter are also, in their essence, nothing else than condensations of the electromagnetic field, our present view of the universe presents two realities which are completely separated from each other conceptually, although connected causally, namely, gravitational aether and electromagnetic field, or as they might also be called space and matter (Einstein, 1920).

Einstein identified the gravitational aether with the static gravitational field and this field with the spacetime that he called the metric field... of who arises physical properties (Cassini and Levinas, 2009). In this context the author observes superfluous the word aether. Also, maybe, Einstein reintroduced aether motivated by his most close colleagues, Lorentz, Weyl and specially Eddington his influential promoter. The author considered probably that they did not believe sufficiently in the geometric explication on gravity of general relativity. In 1916, likely Lorentz influenced in the sudden change of Einstein, accepting the ether. In 1918, Weyl said: the metric field, described by the coefficients guv is more aether that gravitational field and, in 1920, before of the conference of Einstein in Leyden, Eddington declared similarly to Weyl. Clearly, the concept of aether, from luminiferous aether was changed to electromagnetic aether and from this was changed to relativistic aether. Of other hand, Lorentz and other scientists as Michelson and Heaviside, continued defending the ether until their last days, to end of decade 1920. During the next decade of 1930, the word aether fell into disuse. The scientists changed the concept of aether by the concept of field, used currently. Einstein abandoned the concept of aether in 1938 (Cassini and Levinas, 2009).

Between 1916 and 1934, Einstein stated that for physical reasons, gravity, spacetime is the static gravitational field which in turn is determined by physical factors. Of course, there is a symmetrical relationship between geometry and physics, since for physical reasons arises geometry and of this arises physical properties. Regarding the relationship, in the sense from physics to geometry Einstein said: On physical grounds it was assumed that the metrical field was at the same time the gravitational field... Since the gravitational field is determined by the configuration of masses and changes with it, the geometric structure of this space is also dependent on physical factors... Since the gravitational field is determined by the configuration of masses and changes with it, the geometric structure of this space is also dependent on physical factors. 
Thus, according to this theory space is exactly as Riemann guessed no longer absolute; its structure depends on physical influences. (Physical) geometry is no longer an isolated self-contained science like the geometry of Euclid (Einstein, 1934).

In 1938, once again Einstein suddenly changed his concept of the gravity as aether and, he said: this is the moment to forget the ether completely and to try never to mention its name. We shall say: our space has the physical property of transmitting waves, and so omit the use of a word we have decided to avoid (Einstein and Infeld, 1938). Although it could interpret, this declaration, as a methodological stop to return to the aether with definitive arguments, absolutly it was said with the goal of abandon such concept, since this was occurred.

In effect, the concept of aether was changed by the concept of field but this time aether disappeared of the normal science. In 1954, proximate to pass away, Einstein returns to his original thesis of the general relativity, final version of 1916, the spacetime has not existence independent of the field (matter-energy?). He specified that the gravitational field are the functions $g_{u v}$, i.e, geometric and causal relations between events (such as distance, volume, curvature, angle, present, past and future) inducing to think that spacetime is structural property of a geometric field, like Minkowski's spacetime, consistent with general relativity, therefore static gravitational field is a geometric field, since he declared: In order to be able to describe at all that which fills up space and is dependent on the co-ordinates, space-time or the inertial system with its metrical properties must be thought of at once as existing, for otherwise the description of "that which fills up space" would have no meaning. On the basis of the general theory of relativity, on the other hand, space as opposed to "what fills space", which is dependent on the co-ordinates, has no separate existence. Thus a pure gravitational field might have been described in terms of the $g_{\mathrm{uv}}$ (as functions of the co-ordinates), by solution of the gravitational equations. If we imagine the gravitational field, i.e. the functions $g_{\mathrm{uv}}$, to be removed, there does not remain a space of the type (1) (tipe 1 is Minkowski's spacetime), but absolutely nothing, and also no "topological space". For the functions guv describe not only the field, but at the same time also the topological and metrical structural properties of the manifold... A space of the type (1), judged from the standpoint of the general theory of relativity, is not a space without field, but a special case of the $g_{\mathrm{uv}}$ field, for which - for the co-ordinate system used, which in itself has no objective significance - the functions $g_{\mathrm{uv}}$ have values that do not depend on the co-ordinates. There is no such thing as an empty space, i.e. a space without field... Space-time does not claim existence on its own, but only as a structural quality of the field... there exists no space "empty of field" (Einstein, 1954). Thus, Einstein philosophically returned to the relationism.

\section{SPACETIME}

However, the final position of Einstein on space time is in terms of he, but not for the science. Return us to the historic development of field that Einstein declared like the ontological immediate support of the spacetime. At the time that the fields were introduced, it was said: It is characteristic of the fields mentioned that they occur only within a ponderable mass; they serve only to describe a state of this matter... where no matter was available there could also exist no field... when light was regarded as a wave-field, completely analogous to the mechanical vibration field in an elastic solid body. It was thus felt necessary to introduce a field, that could also exist in "empty space" in the absence of ponderable matter... in accordance with its origin, the field concept appeared to be restricted to the description of states in the inside of a ponderable body... that every field is to be regarded as a state capable of mechanical interpretation, and this presupposed the presence of matter. One thus felt compelled, even in the space which had hitherto been regarded as empty, to assume everywhere the existence of a form of matter, which was called "aether"... in connection with the researches of Faraday and Maxwell it became more and more clear that the description of electromagnetic processes in terms of field was vastly superior to a treatment on the basis of the mechanical concepts of material points. By the introduction of the field concept in electrodynamics, Maxwell succeeded in predicting the existence of electromagnetic waves... As a result of this, optics was, in principle, absorbed by electrodynamics. Nevertheless, it was at first taken for granted that electromagnetic fields had to be interpreted as states of the aether... But as these efforts always met with frustration, science gradually became accustomed to the idea of renouncing such a mechanical interpretation... The aether-theory brought with it the question: How does the aether behave from the mechanical point of view with respect to ponderable bodies? Does it take part in the motions of the bodies, or do its parts remain at rest relatively to each other? Many ingenious experiments were undertaken to decide this question. The following important facts should be mentioned in this connection: the "aberration" of the fixed stars in consequence of the annual motion of the earth, and the "Doppler effect", i.e. the influence of the relative motion of the fixed stars on the frequency of the light reaching us from them, for known frequencies of emission. The results of all these facts and experiments, except for one, the Michelson-Morley experiment, were explained by $\mathrm{H}$. $\mathrm{A}$. Lorentz on the assumption that the aether does not take part in the motions of ponderable bodies, and that the parts of the aether have no relative motions at all with respect to each other. Thus the aether appeared, as it were, as the embodiment of a space absolutely at rest... Concerning the experiment of Michelson and Morley, H. A. Lorentz showed that the result obtained at least does not contradict the theory of an aether at rest. In spite of all these beautiful successes the state of the theory was not yet wholly satisfactory, and for the following reasons. Classical mechanics... teaches the equivalence of all inertial systems or inertial "spaces" for the formulation of natural laws... Electromagnetic and optical experiments taught the same thing with considerable accuracy. But the foundation of electromagnetic theory taught that a particular inertial system must be given preference, namely that of the luminiferous aether at rest. This view of the theoretical foundation was much too unsatisfactory... like classical mechanics, would uphold the equivalence of inertial systems (special principle of relativity)? The answer... is the special theory of relativity... The whole content of the special theory of relativity is included in the postulate: The laws of Nature are invariant with respect to the Lorentz transformations. (Einstein, 1954). Therefore the result is the superfluous aether and obviously relevant the original conception of the field as a theoretical tool for the description of the states of matter, direction followed by general relativity, since spacetime is the structural property of static gravitational field, and static gravitational field is the metric field, that through of the Einstein's equations, describes the geometric of Matter. 
Contrary to general relativity based in the general covariance (general equivalence principle between inertial system, accelerated system and gravitational system) and in the mathematical development of tensorial differential geometry, when Einstein both introduced the relativistic ether as when he replaced it by the metric field, he it supported only in new arguments, i.e in the reflective interpretation of his past works. Also, free of gravity of the mathematics, when Einstein adopted the field, he expressed hidden tearing that was dwelling in their thoughts: How could have physical effects, the geometric gravity? And clearly, he declared his intentions by quantum gravity, unified with electromagnetic field, from the vision of material phenomena, presenting arbitrarily the following hypothesis, inside of a vision of future: Our first attempts to go beyond the mechanical view and to introduce field concepts proved most successful in the domain of electromagnetic phenomena. The structure laws for the electromagnetic field were formulated; laws connecting events very near to each other in space and time... Later the general relativity formulated the gravitational laws. Again they are structure laws describing the gravitational field between material particles... We have two realities: matter and field. There is no doubt that we cannot at present imagine the whole of physics built upon the concept of matter... For the moment we accept both the concepts. Can we think of matter and field as two distinct and different realities? Given a small particle of matter, we could picture in a naive way that there is a definite surface of the particle where it ceases to exist and its gravitational field appears. In our picture, the region in which the laws of field are valid is abruptly separated from the region in which matter is present. But what are the physical criterions distinguishing matter and field? Before we learned about the relativity theory we could have tried to answer this question in the following way: matter has mass, whereas field has not. Field represents energy, matter represents mass... From the relativity theory we know that matter represents vast stores of energy and that energy represents matter. We cannot, in this way, distinguish qualitatively between matter and field, since the distinction between mass and energy is not a qualitative one. By far the greatest part of energy is concentrated in matter; but the field surrounding the particle also represents energy, though in an incomparably smaller quantity. We could therefore say: Matter is where the concentration of energy is great, field where the concentration of energy is small... There is no sense in regarding matter and field as two qualities quite different from each other. We cannot imagine a definite surface separating distinctly field and matter. The same difficulty arises for the charge and its field. It seems impossible to give an obvious qualitative criterion for distinguishing between matter and field or charge and field. Our structure laws, that is, Maxwell's laws and the gravitational laws, break down for very great concentrations of energy or, as we may say, where sources of the field, that is electric charges or matter, are present... the division into matter and field is, after the recognition of the equivalence of mass and energy, something artificial and not clearly defined. Could we not reject the concept of matter and build a pure field physics? What impresses our senses as matter is really a great concentration of energy into a comparatively small space. We could regard matter as the regions in space where the field is extremely strong... Its final aim would be the explanation of all events in nature by structure laws valid always and everywhere. A thrown stone is, from this point of view, a changing field, where the states of greatest field intensity travel through space with the velocity of the stone. There would be no place, in our new physics, for both field and matter, field being the only reality. This new view is suggested by the great achievements of field physics, by our success in expressing the laws of electricity, magnetism, gravitation in the form of structure laws, and finally by the equivalence of mass and energy. Our ultimate problem would be to modify our field laws in such a way that they would not break down for regions in which the energy is enormously concentrated. But we have not so far succeeded in fulfilling this programme convincingly and consistently. The decision, as to whether it is possible to carry it out, belongs to the future. At present we must still assume in all our actual theoretical constructions two realities: field and matter. Fundamental problems are still before us. We know that all matter is constructed from a few kinds of particles only. How are the various forms of matter built from these elementary particles? How do these elementary particles interact with the field? By the search for an answer to these questions new ideas have been introduced into physics, the ideas of the quantum theory. (Einstein and Leopold, 1938). In this hypothesis, deliberately ignored by the normal science, Einstein considered the static gravitational field different to geometric field, like physical field, compound by quantas. Then, the mathematical development of the general relativity would be different to current theory, and the general relativity would be a physical theory on gravity, i.e, a theory different to geometric general relativity.

Objectively, the logical chain: Matter $\leftrightarrow$ geometric and causal relations between material events $\leftrightarrow$ metric field $\leftrightarrow$ static gravitational field $\leftrightarrow$ curved spacetime $\leftrightarrow$ gravity, of the thesis of the general relativity, that is a relational theory, in ontological terms, relativity presents: Matter and spacetime like a category of thinking, i.e gravity is a ¡category of thinking $i$, however, with physical effects.

In effect: We are able to produce a gravitational field merely by changing the system of coordinates (Einstein, 1916). According to the general relativity theory, gravitation thus plays an exceptional role as distinguished from the others, specially the electromagnetic forces, in as much as the 10 functions guv representing gravitation, define immediately the metrical properties of the four-dimensional region (Einstein, 1916). That in general, Laws of Nature are expressed by means of equations which are valid for all co-ordinate systems, that is, which are covariant for all possible transformations. It is clear that a physics which satisfies this postulate will be unobjectionable from the standpoint of the general relativity postulate. Because among all substitutions there are, in every case, contained those, which correspond to all relative motions of the co-ordinate system (in three dimensions). This condition of general covariance which takes away the last remnants of physical objectivity from space and time, is a natural requirement, as seen from the following considerations. All our well-substantiated space-time propositions amount to the determination of space-time coincidences. If, for example, the event consisted in the motion of material points, then, for this last case, nothing else are really observable except the encounters between two or more of these material points. The results of our measurements are nothing else than well-proved theorems about such coincidences of material points, of our measuring rods with other material points, coincidences between the hands of a clock, dial-marks and point-events occurring at the same position and at the same time. (Einstein, 1916). Time and space and gravitation have no separate existence from matter...Physical objects are not in space, but these objects are spatially extended. In this way the concept 'empty space' loses its meaning. (Einstein, 1950). 
Inevitably, in the general relativity, gravity is linked strongly with spacetime. Both, spacetime and gravity gnoseologically are categories of the thinking, although these are indirect classifications, necessarily induced, since that general relativity lacks of the explicit conceptual definition of spacetime but whether of gravity like the curvature of spacetime. Spacetime like structural property of gravitational field is not a conceptual definition on it, but whether a specification on it, like also is other specification, its contingent nature, however, they can be elements to define it.

In the general relativity, spacetime is defined operationally, like Newton did with gravity, given that his explanation on gravity, as effect of the hit on the bodies, of the corpuscles of the gravitational aether, no it considers valid. Spacetime is mathematically defined, like a mathematical model that, of a physical dynamic system, combines space and time into a manifold of four dimensions. Space is the three dimensional continuous, that by coordinates $\mathrm{x}_{1}, \mathrm{x}_{2}, \mathrm{x}_{3}$, geometrically represents the place occupied by the universal set of bodies (substantialist model), or constructed by universal set of relations between bodies (relationist model), in geometric terms, the bodies have relative position, direction and sense. Time is the one dimension, that through the coordinate, $x 4$, represents the instant in that the events occur, placed in time as order of succession, according substantialist model or non distinct from things existing in time, according relationist model, in geometric terms, events has relative order of past, present and future. Thus, in the substantialist model: All things are placed in time as to order of succession; and in space as to order of situation (Newton, 1729). Or in the relationist model: Space to be something merely relative, as time is. Space is an order of coexistences, as time is an order of successions (Leibnitz, 1717).

Surely, as spacetime is not physically defined, correspond do it, to philosophy of science. Although the general relativity is originally a relational theory based in the geometric and causal relations between material events, since in general relativity spacetime is not compound of inmaterial points as in Newton, and spacetime as category of the thinking, according to philosophy corresponds its study to metaphysics, but the philosophy of science is who has elaborated the relationist theory. Also to the metaphysic corresponds the spacetime considered like a inmaterial substance according to the philosophical rival conception, in this case, the philosophy of science has elaborated the substantivalist theory, and metaphysics, the theory of super substantivalism. These two philosophical theories, of relationism and substantivalism, arise of old ontological discussion on nature of spacetime, since spacetime as problem that must resolve the philosophy transcends to general relativity, but due mainly to that, this theory has various mathematical models and experimental measurements about the symmetric physical effects of spacetime on matter and matter on spacetime, surely lead to spacetime like a substance, since spacetime acts on matter giving it form, causing it moves within geodesics and producing the effects of the gravitational lense, the dilation of time, the gravitational redshift, the Shapiro time delay and the geodetic precession; of other hand, the matter acts on the space-time, curving it, twisting it, dragging it and dynamically wavy its curvature (Guillen, 2013). Surely, the profound reason to take the spacetime like a substance is not argument of sophisticated substantivalism on the inertial real structure, but due to the symmetric real interactions between spacetime and Matter derived of general relativity.

The unsustainable intellectual tension between relationism and substantivalism, that in the case of Einstein triumphed finally the relationism, in change, paradoxically, in the arena of the philosophy, inside of the context of general relativity, was won by the substantivalism, first with the sophisticated substantivalism, lastly with the super substantivalism (Guillen, 2013). This is the consensus of most scientists. The reason for that in the general relativity, inherited of the classical physics, it conserves the medieval philosophical discussion between Leibnitz and Newton on spacetime, is due to the absence of its physical definition.

Course, the solution of the theory of substantivalism, on spacetime transforms the general relativity in other theory, since originally general relativity is a relationist theory, although the solutions to vacuum of the Einstein's equations allow a substantivalist interpretation. However, such transformation is physically supported in that in general relativity from geometric relations between material events (since metric captures all the geometric and causal structure of spacetime) functions $\mathrm{g}_{\mathrm{uv}}$, are producing physical effects. But, truly it is a Pyrrhic victory of substantivalism, inasmuch as: How, can act metaphysical spacetime on physical Matter?. How, can act physical Matter on metaphysical spacetime?. Worse is the result of super substantivalism, that from general relativity, the material being becomes geometric being, since material being becomes spacetime, i.e geometric spacetime is evaporating the material reality in geometry (Guillen, 2013).

\section{VACUUM}

If substantivist hypothesis were true, then the naked spacetime would exist, i.e. the absolute vacuum, therefore, the spacetime could be observed directly. But, non there is absolute vacuum. Universe is vacuum, matter and radiation. They are material forms of existence, by being objectives and, have physical reality, in last instance, they are the Matter.

Vacuum is a medium that permeates totally the Universe, mainly the called outer space, i.e the space almost totally emptiness between stars, where the density is $10^{-24} \mathrm{~g} / \mathrm{cm}^{3}$, and in the Universe $10^{-30} \mathrm{~g} / \mathrm{cm}^{3}$ (Marquardt, 1999). Also, atoms are mostly empty space, more than 99,999 percent. This means that Matter is mostly vacuum.

In 1916, Nernst based in the quantum theory and Planck's law for the radiation from a black body, proposed that the vacuum is not 'empty' but is a medium filled with radiation which contains a large amount of energy. In the mid-late 1920s, with the developments in quantum electrodynamics (QED), the energy density of the vacuum acquired credibility, since the electromagnetic field is treated as a collection of quantized harmonic oscillators, and contrary to a classical harmonic oscillator, which can be completely at rest and have zero energy, each quantized harmonic oscillator has a non-vanishing 'zero-point' energy. Also, Pauli pointed about the gravitational effects of such zero-point energy. In the equations of general relativity, this vacuum energy is represented by the cosmological constant $\Lambda$, which implies an expanding universe. In 1927, Lemaitre constructed a model of the universe with a cosmological constant, and, in 1934, he commented, on this constant, as the energy in vacuo that would be different from zero. In the late 1940s, the ideas of Nernst and Pauli 
connected with the ideas of Dirac (with his hole theory from 1930) and also the final version of QED constructed by Schwinger, Feynman, and others, had not any interest in its possible gravitational consequences. Nevertheless, the cosmological constant was not completely forgotten, as evidenced in a quote from a conference by Bohr. In 1967, Zel'dovich published the first discussion of the contribution of quantum fluctuations to the cosmological constant, but does not address why the zero-point energies of the fields do not build up a huge cosmological constant. So, he assumed, that the zero-point energies, as well as higher order electromagnetic corrections to this, are effectively cancelled to zero. In the next year, Zel'dovich emphasizes that zero-point energies of particle cannot be ignored when gravitation is taken into account. In mid-1970s, Linde, Dreitlein and Veltman first pointed out the connection between cosmology and spontaneous symmetry breaking mechanism invoked in the electroweak theory. In 1977, following these discussions, Bludman and Ruderman argue that the vacuum energy density was very large at the time of the symmetry breaking. In the early 1980s, with the advent of inflationary cosmology, it stimulated further interest in vacuum energy with cosmological effects. It believed that various components of quantum field theories contribute to the vacuum energy density and it is believed to imply a huge cosmological constant. According to the Standard Model, matter is made up of leptons and quarks which are interacting through three basic types of interactions: electromagnetic, weak and strong interactions. Whereas the electromagnetic and weak forces are unified in the electroweak theory (Glashow-Salam-Weinberg theory), the theory of strong interactions, quantum chromodynamics, comprises a sector of its own. The Standard Model includes an additional coupling of its constituents fields to Higgs fields which play a crucial role both in constructing the electroweak theory, and in generating the masses of the Standard Model particles. The energy estimates for the ground state (the vacuum state) of the Standard Model are in terms of individual vacuum contributions. The vacuum energy density receives contributions from any quantum fields which may exist (Rugh and Zinkernagely, 2000).

Thus, according to general assumptions of quantum physics and quantum field theory, the vacuum in the Universe is filled with a collection of quantum fields, in special with low-energy electromagnetic waves, random in phase and amplitude and propagating in all possible directions. Exists a called vacuum state, inside the region of space, of lowest possible energy which can be reached giving the evolutionary boundary conditions of the physical system. But may correspond to a local minimum of energy, since exist two states of vacuum according its energy. One is the true vacuum, that is the lowest minimum in energy and the other is false vacuum that correspond to one of the other minima. When does it have absolute lowest minimum energy? Taking all the states? If exist two minima energy local vacuum states separated by a potential barrier, they can remain almost an unlimited time, since there is not flux between them, but if the transition from false vacuum, to the true vacuum is triggered then, there would be an explosion. In classical mechanic a particle has the lowest energy when it is resting at the bottom of the potential well. In quantum mechanics the particle in the state lowest energy carries out a zero-point motion. Therefore, everywhere space exhibits zero-point fluctuations, even in regions which are otherwise 'empty' (i.e. devoid of matter and radiation). These zero-point fluctuations of the quantum fields, as well as other 'vacuum phenomena' of quantum field theory, give rise to an enormous vacuum energy density. (DeWitt, 1967, Rafelski and Muller, 1985, Davis and others, 2006, Oldershaw, 2009).

In 1948, experimentally was confirmed in the Philips laboratories, the vacuum energy effect that Casimir predicted. The Casimir energy is a pure vacuum energy; real particles are not involved, only virtual particles (DeWitt, 1996). The Casimir effect is a small attractive force which acts between two closed parallel uncharged conducting plates. It is due to quantum vacuum fluctuation of the electromagnetic field. All fields, in particular electromagnetic fields, have fluctuations. This effect proves that the vacuum is not really empty. It is filled with virtual particles, which are in a continuous state of fluctuation. Virtual particle-antiparticle pair can be created from vacuum and annihilated back to vacuum. These virtual particles exist for a time dictated by Heisenberg uncertainty relation: $\Delta \mathrm{E} . \Delta \mathrm{T} \approx \hbar$. Virtual photons (quanta of electromagnetic waves) are the dominant virtual particles in vacuum fluctuations but other particles produced as well. As vacuum is as a superposition of many different states of electromagnetic field, the creation and subsequent absorption of a photon by the vacuum implies the vacuum fluctuates. (Nguyen, T. 2003).

However, the quantum theory on vacuum presents some problems, the main problem is the called cosmological constant problem, due to that the predictions, about the energy density, do not coincide with the experiments and the Casimir effects can be formulated and Casimir forces can be computed without reference to zero-point energies by relativistic, quantum forces between charges and currents (Saunders, 2002 and Jaffe, 2005). But in such case the naked spacetime would exist, and it would be a iphysical entity i since it has physical properties, an absurd cuestion, also speed of light in empty space would be infinitum. Other problem is whether the vacuum gravitates, surely due to equivalence between energy and mass, nonetheless there is a school of physicists that asserts the energy of vacuum does not carry mass.

\section{CONCLUSIONS}

The result, of this work, is that general relativity lacks of physical definition of spacetime and general relativity leads to philosophical definitions on spacetime that are unacceptable physically. And in summary are:

- As spacetime does not exist naked necessarily is contingent.

- But, spacetime is not structural property of a geometric field, an ideal geometric thing.

- Therefore, spacetime necessarily is a property of the Matter, like a state, in the original sense of field.

- Gravity is not the curvature of the spacetime, since spacetime is a property of the Matter.

- Since static gravitational field is not a geometric field, therefore is a material field, surely similar to static electromagnetic field. If it considers, the static gravitational field like geometric field leads to absurd medieval 
theories, similar to the dualist theory of Descartes on soul, since he said, that the inmaterial soul acts on material body through of the pineal gland, sitting in the sella turcica, a bone of the skull.

- $\quad$ Aether does not exist, but vacuum exist.

- Vacuum is a quantum medium, that permeates totally the Universe.

- The vacuum has similar physical properties to luminiferous aether in the sense that transports the electromagnetic wave and to gravitational aether in the sense, that must transport the static gravitational action, like it was intended by who had believed in the gravitational aether.

- Gravity necessarily is a quantum phenomenon, very likely acting on vacuum, and independent of spacetime.

\section{REFERENCES}

Aristotle. (1930). Physics. Book IV. Works of Aristotle v. 2,Tr Hardie R.P. and Gaye: R.K. W.D. Ross, ed. (Oxford: Clarendon Press)

Baird, E. (2000). Newton's aether model:arXiv

Cassini, A and Levinas, M. (2009). El éter relativista: un cambio conceptual inconcluso:Crítica, revista Hispanoamericana de filosofía. Vol 41, No 123

Dannon, V. (2011). The Meaning of the Michelson-Morley Experiment

Davis, E, Teofilo, V, Haisch, B, Puthoff, H, Nickisch, L, Rueda, A and Cole, D. (2006). Review of Experimental Concepts for Studying the Quantum Vacuum Field:Space Technology and Applications International Forum

Descartes, R. (1969). The Essential Descartes: Edited by Margaret D. Wilson, Mentor Books

DeWitt, B. (1967). Quantum theory of gravity. I. The canonical theory:Phys. Rev. 160, 1113-1148

DeWitt, B. (1996). The Casimir Effect in Field Theory:A. Sarlemijn and M. J. Sparnaay, 247-272

Eddington, A. (1938). The philosophy of physical science:Tarner Lectures

Einstein, A. (1905a). On the electrodynamics of moving bodies:collection of the Annus Mirabilis papers

Einstein, A. (1905b). On a Heuristic Point of View Concerning the Production and Transformation of Light:American Journal of Physics, v. 33, n. 5

Einstein, A. (1905c). Does the inertia of a body depend upon its energy content?:collection of the Annus Mirabilis papers

Einstein, A. (1909). On the Development of Our Views Concerning the Nature and Constitution of Radiation:The information Philosopher

Einstein, A. (1916). The Foundation of the General Theory of Relativity:Princeton University Press

Einstein, A. (1917). On the Quantum Theory of Radiation:The information Philosopher

Einstein, A. (1918). Dialog about Objections against the Theory of Relativity:wikisource

Einstein, A. (1920). Aether and the Theory of Relativity:University of Leyden, Germany

Einstein, A. (1934). The problem of space, ether, and the field in physics:Mountain Man Graphics

Einstein, A. (1936). Physics and reality:Hosted by Professor Milivoje M. Kostic

Einstein, A and Infeld L. (1938). The Evolution of Physics: The Growth of Ideas from Early Concepts to Relativity and Quanta:Osmania University

Einstein, A. (1950). On the Generalized Theory of Gravitation:Scientific American

Einstein, A. (1954). Relativity and the Problem of Space:Relativitybook.com

Gower, B. (2002). Scientific Method:Taylor \& Francis e-Library

Guillen, A. (2010). Spacetime geometric structural property of the matter in motion:Petrov 2010 Anniversary Symposium On General Relativity and Gravitation

Guillen, A. (2013). Gravity, metaphysics or physics?:International Journal of Fundamental Physical Sciences, Vol 3, No 4, 68-74

Haldane, A. (2011). A History of aether:Rutgers SSPAR

Jaffe, R. (2005). Casimir effect and the quantum vacuum:Physical review $D$ 72,021301(R)

Jousten, K. (2008). The History of Vacuum Science and Vacuum Technology:Wiley-VCH

Leibnitz, G. (1717). Mr. Leibnitz's Third Paper. Being An Answer to Dr. Clarke's Second Reply.Mathematical 
Principles of Natural Philosophy: University of Sussex, East Sussex. newtonproject@sussex.ac.uk

Marquardt, N. (1999). Introduction to the principles of vacuum physics:CERN-99-05

Newton I. (1729). The Mathematical Principles of Natural Philosophy: Philosophy Archive marxists.org

Newton, I. (1730). Opticks:Dover Publications, INC

Nguyen, T. (2003). Casimir Effect and Vacuum Fluctuation:Spring

Oldershaw, R. (2009). Towards A Resolution Of The Vacuum Energy Density Crisis:arxiv.org

Rafelski, J and Muller, B. (1985). The structured vacuum:Deutsch Publisher

Ranzan, C. (2010). The history of the aether theory:.CellularUniverse.org 3

Rugh, S. E and Zinkernagely, H. (2000). The Quantum Vacuum and the Cosmological Constant Problem:arXiv

Saunders, S. (2002). Is the Zero-Point Energy Real?:World Scientific

Stokes, G. (1845). On the Aberration of Light:Philosophical Magazine, Volume 27, 9-15

Wang, S. (2007). On the super-fluid property of the relativistic physical vacuum medium and the inertial motion of particles: arXiv 